\title{
PAST AND PRESENT STUDIES RELATING TO THE ILLUMINATION OF THE NIGHT SKY
}

\author{
MERLE F. WALKER \\ Lick Observatory Board of Studies in Astronomy and Astrophysics, \\ University of California, Santa Cruz, California 95064
}

During the period from 1965-1977, I was engaged in a program of selecting and acquiring a new dark sky site in California for the Lick Observatory. Even though the effort was, in the end, unsuccessful, this work did lead to several important advances in our understanding of the general problem of optimizing optical-region astronomical observing conditions. These included:

1) The development of the polar star trail method of seeing measurement (Harlan and Walker 1965; Walker 1971).

2) Definition of the conditions required to optimize the seeing through the use of the polar star trail method of measurement at a number of potential and established observing sites around the world (Walker 1970, 1971, 1983, 1984; McInnes and Walker 1974).

3) Demonstration of the widespread effects of atmospheric pollution in reducing photometric quality at potential sites in California (Walker 1970).

4) The first extensive study of the effects of light pollution on astronomical observations.

It is these latter studies, and their continuation to the present time, that I shall discuss here.

That some observatories were being adversely affected by urban growth and consequent increasing light and air pollution had been recognized for many years. Mount Wilson, for example, located at a horizontal distance of about 8 miles from the center of Pasadena, had, even by the early 1940's, undergone such an increase in sky illumination that work on faint objects was no longer possible. And by the early 1960's, the increasing brightness of the night sky at Mount Hamilton led to the need to establish a new dark sky site for the observatory. However, it was not until work on the California Site Survey began that the extent and seriousness of this problem became evident.

On the basis of measures of the sky illumination at Palomar Mountain produced by the lights of San Diego and Los Angeles and of the illumination produced by the lights of San Jose at different distances from that city, and assuming that the light produced by a city was proportional to the number of its inhabitants, it was possible to map the areas (in the southern half of California) that were already subject to an artificial increase in sky brightness of $0.2 \mathrm{mag}$ at an altitude of $45^{\circ}$ in the direction of the city. This, in 1965, was the amount of artificial illumination observed at Palomar due to the lights both of San Diego 
and Los Angeles, and was adopted as the maximum light pollution acceptable at a site for a new observatory. This mapping process showed that already large areas of California were no longer suitable for new dark sky optical observatories due to light pollution (Walker 1970). This mapping was later extended to all of California and Arizona, using improved census figures for the populationdistance relation (Walker 1973). Additionally, in the early 1970's, U.S. Air Force satellite photographs of the nighttime city lights became available and these confirmed the results of the mapping based on the photometric observations (Walker 1973). Together, this material showed very clearly that light pollution had become a very serious threat to ground-based dark sky optical astronomy, and that action by the astronomical community was urgently needed to preserve the capability for faint-object observation in the future. The world-wide nature of the light pollution problem is emphasized by the poster showing nighttime satellite photographs of city lights worldwide exhibited by Dr. Crawford at this Colloquium.

Once the extent and importance of the light pollution problem had been shown, more extensive studies of the sky brightness were carried out using sky brightness photometers designed by Dr. A. W. Whitford (Walker 1970, 1973, 1977). From these studies, an improved brightness distance relation was derived. First, the variation of artificial night sky brightness with distance from the city was determined, using for this purpose the lights of Salinas, since it has a fairly high light output, is concentrated, and is fairly well isolated from other bright cities, so that the effect of its lights can be traced for a considerable distance without interference from other sources. The observed brightness-distance relation is non-linear, but over a reasonable range of distance $(11 \mathrm{~km} \leq \mathrm{D} \leq 100$ $\mathrm{km}), \mathrm{I} \propto \mathrm{D}^{-2.5}$ (Walker 1977). Next, data were obtained from various California cities on their populations and the total luminous output of their street lighting systems. These data confirmed that in fact the luminous output of cities is directly proportional to the number (P) of their inhabitants (Walker 1977). From these results, an improved population-distance relation was derived, showing how far one must be from a city of a given size to have $\leq 0.2 \mathrm{mag}$ of urban light at $45^{\circ}$ altitude toward the city. This distance varies as $\mathrm{P}^{0.4}$ and for a city of $P=1 \times 10^{6}, D \simeq 100 \mathrm{~km}$ (60 miles) (Walker 1977).

More recently, much more elaborate modeling of the effect of the city lights has been carried out by Dr. R. Garstang $(1986,1988)$, and very precise predictions can now be made using the techniques that he will discuss in the following paper.

It is to be noted that at Mount Hamilton, measures of the sky brightness from 1965 to 1978 indicate that since the beginning of the large population growth in San Jose and the Santa Clara Valley, in about 1948, the artificial illumination increased at a rate of about $0.065 \mathrm{mag}^{-1}$ in both $V$ and B. Since 1978 , this rate of increase has continued in V, so that the V-band sky brightness at $45^{\circ}$ altitude in the direction of San Jose is now about $V=20.1 \mathrm{mag} \operatorname{arcsec}^{-2}$. In $\mathrm{B}$, the sky brightness in this position appears to have remained relatively constant during the early 1980 's at about $\mathrm{B}=21.5 \mathrm{mag} \mathrm{arcsec}^{-2}$ as a result of the shift in street lighting from mercury vapor to first high- and then low-pressure sodium. However, the available observations suggest that since 1984, the B-band brightness is once again increasing at about the former rate, and is now about $B$ $=21.3 \mathrm{mag}$ arcsec ${ }^{-2}$. Thus, at $45^{\circ}$ altitude towards San Jose, the Mount Hamilton sky is now about $\delta \mathrm{V}=1.8, \delta \mathrm{V}=1.6 \mathrm{mag}$ brighter than the natural 
level, and the effect of changing from mercury to sodium street lighting appears to have been to decrease the B-band sky illumination at $45^{\circ}$ altitude by about 0.3 mag. This relatively small decrease emphasizes the fact that street lighting is far from being the only source of light pollution.

So far, I have discussed the effects of artificial illumination on the night sky. However, at any site natural sky brightness exists as well. This illumination consists on the one hand of extraterrestrial sources such as the zodiacal light and on the other of atmospheric sources such as the air glow and the aurorae. The effects of the extraterrestrial sources will be the same at all sites. The effects of the atmospheric sources may not; the aurorae, for example, are more frequent and more intense at high latitudes.

Since 1980, observations have been carried out at San Benito Mountain in California to investigate the variation of the natural atmospheric sky brightness in the $V$ and $B$ filter bands with the level of solar activity (Walker 1988). These studies show:

1) The brightness of the zenith sky decreases exponentially after the end of astronomical twilight. Most of the observations of this effect cover only the first half of the night, as there is only a short period around March each year when observations can be made at the zenith all night without interference from the Milky Way. However, observations on March 20, 1988 (UT), shown in Figure 1, indicate that the sky brightness continued to decrease until dawn, so that it is in fact true, as we have been told all our lives: "it is darkest just before the dawn." The magnitude of this decrease is substantial, amounting to about $\delta \mathrm{V}=\delta \mathrm{B}=$ 0.4 mag between the end of evening twilight and dawn. Thus, observers attempting to reach the faintest possible limit should observe during the latter part of the night.

2) Correcting for the about "post-twilight decay" in sky brightness, the zenith sky brightness does vary with the level of solar activity. The best correlation exists with the intensity of the $10.7-\mathrm{cm}$ radio flux, which is an indicator of the intensity of solar EUV flux in the wavelength range from $100 \mathrm{~A}$ to $625 \mathrm{~A}$. The amount of the variation with solar activity is quite large. From the observed maximum and minimum values of the $10.7-\mathrm{cm}$ flux, we can expect that the zenith sky brightness will vary as much as $\delta \mathrm{V}=0.96 \mathrm{mag}, \delta \mathrm{B}=0.83 \mathrm{mag}$ as a result of solar activity. This effect must therefore be allowed for, both in assessing the quality of existing or potential observing sites, and in planning observations of the faintest possible sources. At present, little is known about how the broad-band $V$ and $B$ sky brightness may vary with season and/or geographic position. Extensive observations are thus needed as a guide to selecting the darkest worldwide sites for ground-based observations. These observations do not require a large telescope and therefore can be carried out at any observatory using a small, dedicated instrument. A regular program of such observations should be a part of the on-going work at all dark-sky observatories, not only to elucidate the nature of the natural sky brightness variations, but also to monitor changes due to developing urban light-pollution. Such a record is invaluable when undertaking to convince civil authorities of the need to institute lighting controls. 


\section{REFERENCES}

Garstang, R. 1986, Publ. A.S.P., 98, 364. . 1988, Publ. A.S.P., in press.

Harlan, E. A. and Walker, M. F. 1965, Publ. A.S.P., 77, 246.

McInnes, B. and Walker, M. F. 1974, Publ. A.S.P., 86, 529.

Walker, M.F. 1970, Publ. A.S.P., 82, 487. . 1971, Publ. A.S.P., 83, 401. 1973, Publ. A.S.P., 85, 507. 1977, Publ. A.S.P., 89, 405. 1983, Publ. A.S.P., 95, 903..PA

Fig. 1 Zenith sky brightness at San Benito Mountain on March 20, 1988 (UT). Ordinate, sky brightness in magnitudes $\operatorname{arcsec}^{-2}$. Abscissa, time after the end of $18^{\circ}$ morning astronomical twilight. The line marked "Dawn" indicates the time of the beginning of $18^{\circ}$ morning astronomical twilight. The curved lines represent the mean variation of sky brightness with time after the end of twilight observed between 1976 and 1987 at San Benito Mountain, arbitrarily fitted to the present observations.

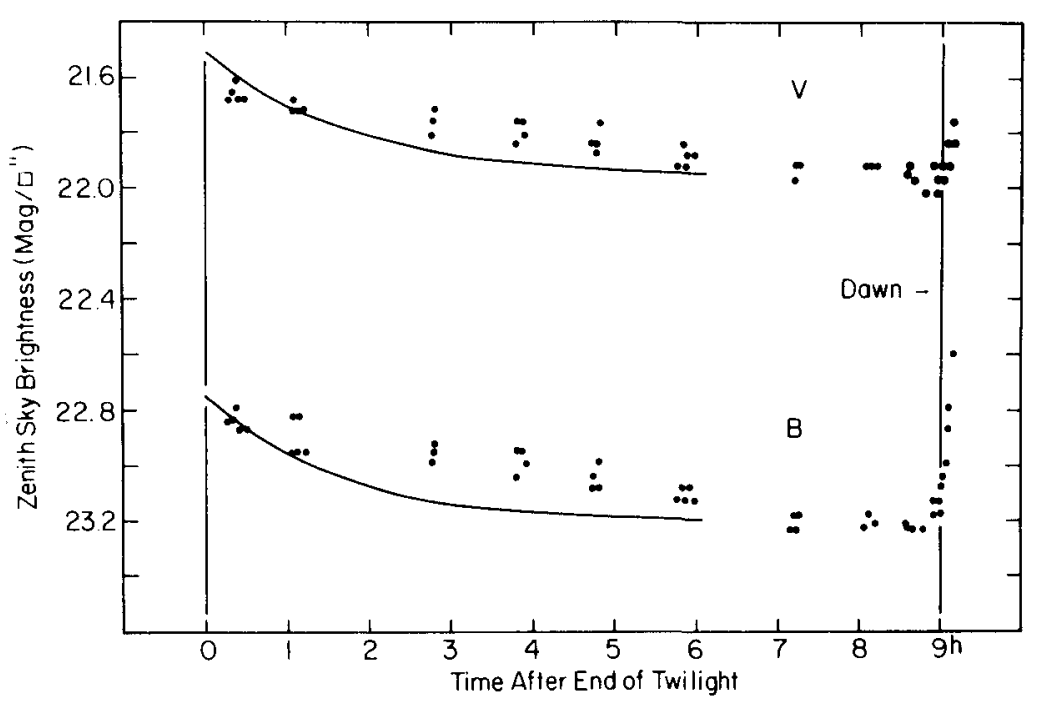

